\title{
Donepezil and Selegiline to Improve Balance Control in Early Progressive Supranuclear Palsy
}

\section{Emmanuelle Pourcher ${ }^{1,2, *}$, Jaime McDonald ${ }^{3,4}$ and Philippe Corbeil ${ }^{4,5}$}

${ }^{1}$ Québec Memory \& Motor Skills Disorders Research Center, Clinique Sainte-Anne, Québec, Canada

${ }^{2}$ Department of Medicine, Faculty of Medicine, Laval University, Québec, Canada

${ }^{3}$ Department of Pharmacy, Children's Hospital of Eastern Ontario, Ottawa, Ontario, Canada

${ }^{4}$ Groupe de recherche en analyse du mouvement et ergonomie, Faculty of Medicine, Kinesiology, Laval University, Québec, Canada

${ }^{5}$ Unité de recherche sur le vieillissement, Centre de recherche FRSQ du Centre Hospitalier affilié Universitaire de Québec, Canada

*Corresponding author: Emmanuelle Pourcher, Québec Memory \& Motor Skills Disorders Research Center, Clinique Sainte-Anne, Québec, Canada, Tel: 1-418-692-2227; Fax: 1-418-692-3338; E-mail: csa@riq.qc.ca

Rec date: Mar 08, 2014; Acc date: Apr 15, 2014; Pub date: Apr 18, 2014

Copyright: ( 2014 Pourcher E. et al. This is an open-access article distributed under the terms of the Creative Commons Attribution License, which permits unrestricted use, distribution, and reproduction in any medium, provided the original author and source are credited.

\begin{abstract}
Background: No single pharmacologic treatment has proven effective for the symptomatic management of progressive supranuclear palsy.

Methods: We report a case of postural instability and falls secondary to PSP of the Parkinson's subtype and initial monotherapy with donepezil $10 \mathrm{mg}$ daily for 3 months followed by a novel investigation of selegiline in combination for 6 additional months. Fall frequency, clinical pull testing and computerized dynamic posturography were used to assess response.
\end{abstract}

Results: After 3 months of donepezil, fall frequency decreased from approximately 5 to 1 per week and an initially pathologic pull-test had normalized. Posturographic improvements occurred with donepezil monotherapy and after 3 and 6 months of combination therapy with selegiline. Clinical improvements were observed over the course of more than one year.

Conclusions: Improvements in clinical and posturographic measures of balance control were observed with donepezil monotherapy and, to a minimal extent, with the addition of selegeline.

Keywords: Progressive supranuclear palsy; Neuropharmacology; Quality of life

\section{Introduction}

Progressive Supranuclear Palsy (PSP) is an atypical parkinsonian syndrome characterized by early falls and non-responsiveness to levodopa [1]. In PSP, neuronal degeneration involves not only the prefrontal cortex and striatonigral structures, but also the thalamus and mesencephalic locomotor area, namely the pedunculopontinecuneiform complex (PPN) [2-3]. This region, known as the major source of cholinergic afferents to the thalamus, has been recently implicated in the control of gait and posture in Parkinson's disease [4-5]. Recent positron emission tomography (PET) studies by Zwergal et al. confirm the functional disturbance of the PPN in PSP and establish a correlation between postural imbalance and falls and the functional pathology of the thalamus [6-7].

There is no standard of care for management of PSP. Reported pharmacological trials have been negative or disappointing, including two trials of donepezil designed to study cognitive endpoints [8-10].

We report a case of postural instability secondary to PSP of parkinsonian subtype (PSP P), which exhibited improvements early in the disease course in response to initial monotherapy with donepezil, and further by combined treatment with selegiline. This combination aimed to increase central cholinergic tone, as well as dopamine and noradrenaline, in a "multiple neurotransmitter" strategy. Experimental data showing a significant decrease in frontal acetylcholine binding in the presence of low noradrenergic tone added support for the combination [11].

\section{Materials and Methods}

Donepezil $10 \mathrm{mg}$ once daily for three months was followed by the addition of selegiline $5 \mathrm{mg}$ twice daily. Computerized dynamic posturography (Pro Balance Master ${ }^{\bullet}$, NeuroCom ${ }^{\circledR}$ International Inc., Clackamas, Oregon, USA) was performed prior to medication, after 3 months of donepezil and after 3 and 6 months of the combination (i.e. at 6 and 9 months). More specifically, to test adaptation of postural responses to repeated support surface displacements, five sets each of toes-up and toes-down platform rotations ( $8^{\circ}$ amplitude, 400 milliseconds) were delivered. Pull test and fall frequency were assessed after 3 months of donepezil, with further evaluation at 9 months (6 months of combination) and again at 18 months as logistical barriers precluded any intermediate evaluation.

Balance recovery was quantified using response latencies and the magnitude and velocity of center of foot pressure (CoP) displacements. The onset latency represents the time interval between the onset of platform movement and the onset of CoP displacement in 
the direction of the loss of balance. The early component of this latency represents the time between the onset of the perturbationevoked balance recovery response until the maximal velocity reached by the CoP. The late component of this latency is defined as the time between the onset of maximal velocity and when CoP velocity returns to zero. A custom-written software program (Matlab 2007b, Mathworks Inc., Natick, MA, USA) was used to compute CoP variables.

A formal review by an institutional ethics committee was not sought as the decision to publish the current case was made retrospectively and both patient and caregiver provided consent for publication of any finding of medication trials.

\section{Case Report, Results and Discussion}

Presented is a 58 -year old male with a 9-month history of motor complaints. Over the preceding year, the family noted a progressive psychomotor slowing with frequent falls as well as a mildly slowed, dysarthric speech and occasional dysphagia. Brain computed tomography was unremarkable and brain magnetic resonance imaging did not reveal sufficient vascular damage to suspect small vessel disease as an etiology. The patient had mild left deafness and complained of dizziness while walking and losing balance when changing position abruptly without support. Otherwise, the patient maintained an active lifestyle. Falls were mostly backward and according to patient and caregiver, averaged at approximately 5 per week in the months leading to diagnosis.

Initial neurological exam was notable for slowness of vertical saccades and square wave jerks on fixation. There was evidence of mild facial, lingual and pharyngeal motor slowness, with bradylalia, aprosody, mild difficulties in coughing maneuvers, as well as mild extra pyramidal rigidity and bradykinesia, without decrement on the left side, without tremor. The patient exhibited abnormal glabellar and snout reflexes, an unsteady tandem walk without lateralization and a pathological pull-test with significant retropulsion and risk of falling backward if unprotected by the examiner. The diagnosis of PSP was confirmed at 18-month follow-up with the appearance of specific supra-nuclear vertical gaze abnormalities.

\section{Clinical improvement was reported by the patient and confirmed by the caregiver:}

After 3 months of donepezil, both patient and caregiver reported a marked decrease in fall frequency to approximately 1 per week. The patient noted transient mild urinary incontinence; no other adverse events were reported. The pull test normalized at 3 months and remained normal at 9 months. With the addition of selegiline, patient and caregiver reported no additional benefit, deterioration or adverse events. At the 18-month evaluation, the pull-test returned to baseline. Clinical deterioration of balance occurred between 15 and 18 months following drug initiation.

\section{Postural improvements were observed at each posturometric evaluation:}

Figure 1 illustrates balance recovery during computerized dynamic posturography for both toes-up (panels $A, C, D, G$ ) and toes-down (panels B,E,F,H) platform rotations at baseline and for each treatment. The initial CoP position taken prior to the onset of platform rotation, as well as the maximal CoP displacement during the perturbationevoked CoP displacements are illustrated in panels C-F. The mean $\mathrm{CoP}$, which is an indicator of overall postural stability during recovery, is described in panels G-H.

As seen in Figure 1, for toes-up rotations only (panel D), the maximum displacement of the CoP after 3 months of donepezil and after 6 months of combination therapy was reduced compared to baseline. This may be important as toes-up platform rotations perturb the center of mass posteriorly, corresponding to the directional preponderance for falls observed in this case. As per panel G, CoP mean velocity improved in both directions following donepezil and after 6 months of the combination, but not after 3 months. For toesdown, results were more consistent across time. Interestingly, CoP velocity in the medial-lateral direction decreased in the toes-down direction, indicative of improved medial-lateral balance control during recovery.

Improvement was perceived by the patient and observed by the caregiver, mainly in response to donepezil, with a clinically significant, progressive reduction in backward falls. This observation supports the role of a cholinergic intervention in postural sensory integration by the thalamus or in attentional cortical mechanisms, under the control of the PPN and the nucleus basalis of Meynert, respectively. Contributions of PPN-thalamic cholinergic projections in postural sensory integration in Parkinson's disease has been recently observed through a correlation between increased postural sway, established as a risk of falling, and reduced thalamic acetylcholinesterase activity [12].

Improvement in prefrontal anticipatory mechanisms is also possible as posturometric improvements occurred mostly in the latter trials at each evaluation; adjustments of preparatory leaning stance are shown in the apparent alteration of the initial position of the CoP across trials. Evidence that effective strategies to minimize perturbation were learned may have implications for physical and rehabilitation therapy.

Improvement of medial lateral balance control during recovery was unexpected; a cholinergic modulation of the cerebellum, which is involved in the refinement of lateral stability, is also possible as it displays connections with the PPN [13].

Our observations, while notable, are difficult to interpret. It is unclear if these improvements represent a true pharmacotherapeutic effect, a placebo response, or a learning effect. The clinician, however, is not accustomed to improvement in dynamic balance in PSP in the first year following diagnosis. The aforementioned negative trial of donepezil reported by Litvan et al. (2001), was conducted in a later stage of PSP (an average of 46 months after diagnosis) and assessed mainly cognitive parameters as opposed to postural outcomes.

The importance of providing beneficial results of individual medication trials, particularly if objective data is available, cannot be overlooked in the absence of an evidence-based approach to PSP treatment. Further investigation into the combined use of acetylcholine, dopamine and noradrenaline modulators to reduce falls in early PSP is warranted. 
Citation: Pourcher E, McDonald J, Corbeil P (2014) Donepezil and Selegiline to Improve Balance Control in Early Progressive Supranuclear

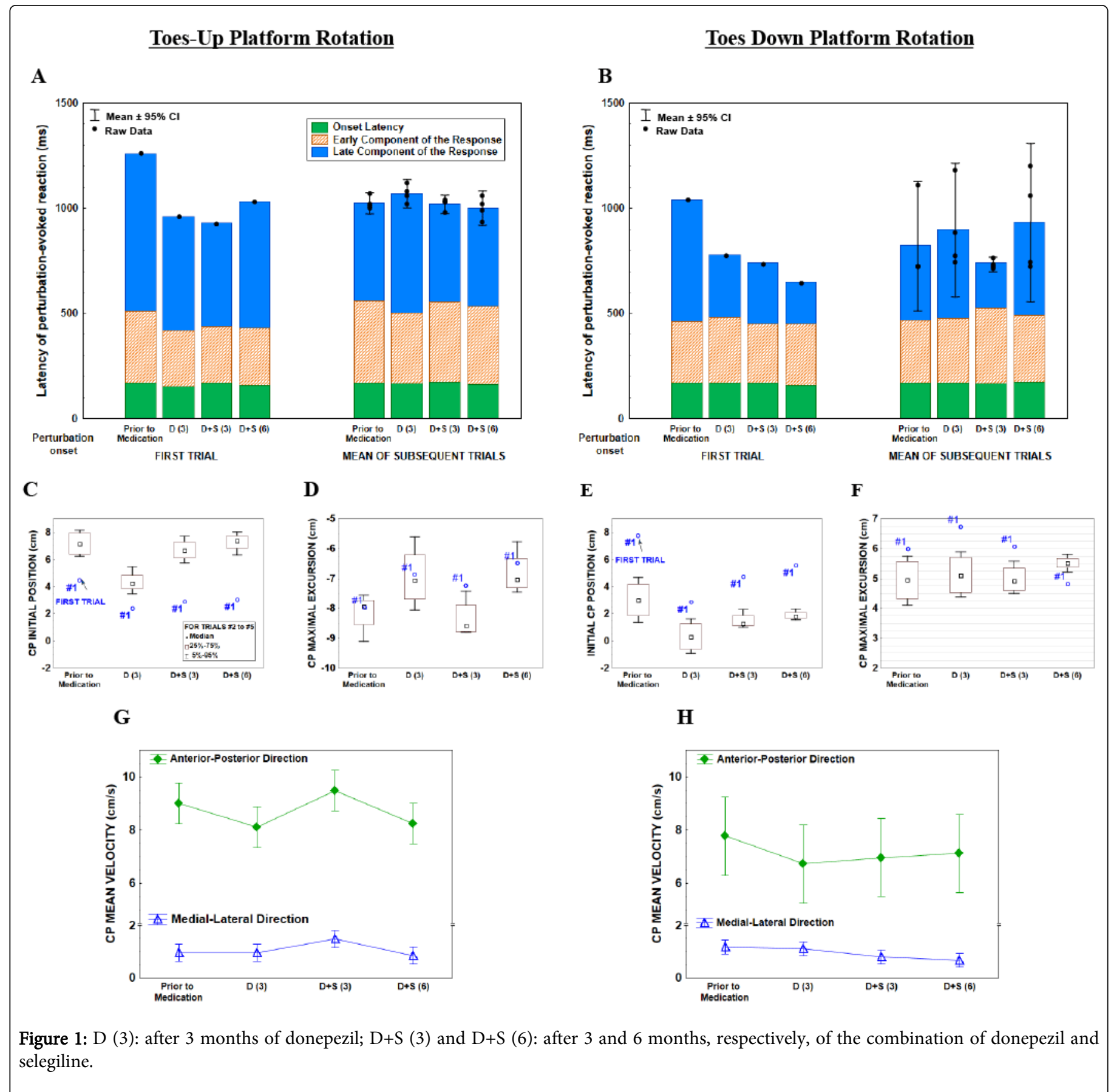

\section{Acknowledgements}

The authors would like to thank the patient and caregiver for their participation in the current trial as well as Yoann Dessery for his assistance in data collection. Dr. McDonald reports grants from les Fonds de la Recherche en Santé du Québec; and sits on the board of directors of the Cancer Advocacy Coalition of Canada (CACC), a notfor-profit advocacy group. Since 2012, the CACC has received grants from Amgen, Boehringer Ingelheim, Eli Lilly, GlaxoSmithKline, Merck, Novartis, Pfizer, Roche, Rx \& D, and Sanofi.

\section{References:}

1. Golbe L (2001) Progressive Supranuclear Palsy. Curr Treat Options Neurol 3: 473-477.

2. Hirsch EC, Graybiel AM, Duyckaerts C, Javoy-Agid F (1987) Neuronal loss in the pedunculopontine tegmental nucleus in Parkinson disease and in progressive supranuclear palsy. Proc Natl Acad Sci U S A 84: 5976-5980.

3. Henderson JM, Carpenter K, Cartwright H, Halliday GM (2000) Loss of thalamic intralaminar nuclei in progressive supranuclear palsy and Parkinson's disease: clinical and therapeutic implications. Brain 123 : 1410-1421. 
Citation: Pourcher E, McDonald J, Corbeil P (2014) Donepezil and Selegiline to Improve Balance Control in Early Progressive Supranuclear Palsy. J Neurol Disord 2: 153. doi:10.4172/2329-6895.1000153

Page 4 of 4

4. Karachi C, Grabli D, Bernard FA, Tandé D, Wattiez N, et al. (2010) Cholinergic mesencephalic neurons are involved in gait and postura disorders in Parkinson disease. J Clin Invest 120: 2745-2754.

5. Bohnen NI, Müller ML, Koeppe RA, Studenski SA, Kilbourn MA, et al. (2009) History of falls in Parkinson disease is associated with reduced cholinergic activity. Neurology 73: 1670-1676.

6. Zwergal A, la Fougère C, Lorenzl S, Rominger A, Xiong G, et al. (2011) Postural imbalance and falls in PSP correlate with functional pathology of the thalamus. Neurology 77: 101-109.

7. Zwergal A, la Fougère C, Lorenzl S, Rominger A, Xiong G, et al. (2013) Functional disturbance of the locomotor network in progressive supranuclear palsy. Neurology 80: 634-641.

8. Fabbrini G, Barbanti P, Bonifati V, Colosimo C, Gasparini M, et al. (2001) Donepezil in the treatment of progressive supranuclear palsy. Acta Neurol Scand 103: 123-125.
9. Litvan I, Phipps M, Pharr VL, Hallett M, Grafman J, et al. (2001) Randomized placebo-controlled trial of donepezil in patients with progressive supranuclear palsy. Neurology 57: 467-473.

10. Nieforth KA, Golbe LI (1993) Retrospective study of drug response in 87 patients with progressive supranuclear palsy. Clin Neuropharmacol 16: 338-346.

11. Acquas E, Wilson C, Fibiger HC (1998) Pharmacology of sensory stimulation-evoked increases in frontal cortical acetylcholine release. Neuroscience 85: 73-83.

12. Müller ML, Albin RL, Kotagal V, Koeppe RA, Scott PJ, et al. (2013) Thalamic cholinergic innervation and postural sensory integration function in Parkinson's disease. Brain 136: 3282-3289.

13. Gilman S, Koeppe RA, Nan B, Wang CN, Wang X, et al. (2010) Cerebral cortical and subcortical cholinergic deficits in parkinsonian syndromes. Neurology 74: 1416-1423. 